\title{
Laser scanning tomography of localised nerve fibre layer defects
}

\author{
Reinhard O W Burk, Anja Tuulonen, P Juhani Airaksinen
}

\begin{abstract}
Aims-Retinal nerve fibre layer photography is a well established method to qualitatively document early structural changes which might be induced by primary open angle glaucoma. The aim was to analyse localised retinal nerve fibre layer (RNFL) defects in a new quantitative way with respect to surface topography, defect width, and surface reflectivity by means of the technique of confocal scanning laser tomography.
\end{abstract}

Methods-12 eyes of 12 patients with a localised RNFL defect documented in RNFL photographs and a normal appearance of the optic disc were enrolled in the study. Using confocal laser scanning tomography (Heidelberg retina tomograph, HRT) a series of 32 optical section images from different focal planes of the retina at the site of the RNFL defects were obtained. The optical section images, the reflectivity images, and the topographic images were analysed regarding the visibility of the RNFL defects. The mean surface height and the reflectance at the sites of the RNFL damage were measured and compared with the adjacent apparently normal retina. The width of the RNFL defect at $1 \mathrm{~mm}$ distance from the disc border was evaluated.

Results-RNFL defects could be detected in nine of 12 reflectivity images (75\%). Single optical section images displayed the RNFL defects in 12 of 12 eyes. The defect width ranged from 0.11 to $1.0 \mathrm{~mm}$. In six of 12 eyes a surface depression (34 (SD 5) $\mu \mathrm{m}$; range $21-47 \mu \mathrm{m}$ ) was present.

Department of Ophthalmology, University of Heidelberg, Germany R O W Burk

Department of Ophthalmology, University of Oulu, Finland

A Tuulonen

P J Airaksinen

Correspondence to: Dr Reinhard O W Burk, Department of

Ophthalmology, University

of Heidelberg, Im

Neuenheimer Feld 400,

D-69120 Heidelberg,

Germany.

Accepted for publication 24 March 1998
The reflectance ratio ranged from 0.68 to 0.94 at the site of the RNFL defect. In eyes with a glaucomatous scotoma in a $6^{\circ}$ grid visual field (VF), the defect width was at least $0.25 \mathrm{~mm}$. Surface depression and low reflectance ratio were found irrespective of the presence of a scotoma in the $6^{\circ}$ grid VF.

Conclusion-The majority of localised RNFL defects can be detected in reflectivity images from laser scanning tomograms. Localised RNFL defects may be differentiated according to surface topography into those with and those without a measurable surface depression. A small but deep RNFL defect is not necessarily associated with a scotoma in routine $6^{\circ}$ grid VF static perimetry. (Br f Ophthalmol 1998;82:1112-1117)
In 1913, Alfred Vogt described the visibility of the nerve fibre bundles by ophthalmoscopy. ${ }^{1}$ Within the past 20 years, the careful clinical evaluation of the retinal nerve fibre layer (RNFL) has become a cornerstone in the diagnosis of early glaucoma. RNFL photography has proved to be superior to other methods in detecting very early glaucomatous optic nerve abnormalities and their progression..$^{2-5}$ RNFL damage may precede visual field defects by several years. ${ }^{67}$

Photographs give two dimensional information of the nerve fibre layer structure which, at least for the time being, is qualitative. Scoring systems have been developed to analyse the defects semiquantitatively. ${ }^{8-10}$ More recently instruments have been designed that are capable of measuring indirectly the thickness of the RNFL at the optic disc margin (Heidelberg retina tomograph, HRT), or provide an estimate of the peripapillary RNFL thickness using the polarisation properties of the axon bundles (nerve fibre analyser, NFA) or other light reflection properties of the RNFL (optical coherence tomograph, OCT).

Owing to observations made with good quality optic disc stereophotograph ${ }^{11}$ and to the development of the aforementioned high technology instruments it is of great practical interest to determine the surface topography and the three dimensional structure of the RNFL at the site of an RNFL defect. We demonstrate in this paper that such measurements have now become possible with the technique of confocal scanning laser tomography.

\section{Methods}

RETINAL NERVE FIBRE LAYER PHOTOGRAPHS In this study, we were interested in examining the two and three dimensional topography of very early RNFL abnormalities. To this end, 12 eyes of 12 patients were selected by two investigators (AT, JPA) based on the presence of localised retinal nerve layer abnormalities in wide angle nerve fibre layer photographs. ${ }^{12}$

Nerve fibre layer photographs had been taken as a routine screening procedure for all glaucoma suspect and glaucoma patients in the department of ophthalmology. The subjects had been referred by their ophthalmologists to rule out the possibility of damage from early primary open angle glaucoma. Four subjects were classified as ocular hypertensive, five were treated because of primary open angle glaucoma ("normal tension glaucoma"), and three patients had relatives in their families who were being treated for manifest glaucoma. The mean age of the patients was 62 years (range $46-73$ years). The $60^{\circ}$ images were taken with 
a Canon CF $60 \mathrm{Z}$ camera on a black and white, low sensitivity, high resolution film (Kodak TMAX 100) using a blue narrow band interference filter of $495 \mathrm{~nm}$ wavelength. The film was developed in Agfa "Refinal" developer at $20^{\circ} \mathrm{C}$ for 10 minutes. Prints were made on Agfa "Brovira" paper, grade 4. The picture quality ranged from excellent to poor as assessed from the RNFL photographs. Images were judged to be excellent if the course of arcuate nerve fibre bundles and small vessels could be easily visualised. In poor images even the large vessel trunks were more difficult to see because of media opacities.

VISUAL FIELD TESTS

The visual fields were obtained using computerised static threshold perimetry (Humphrey 30-2). All patients had at least three visual field examinations, learning fields not included. The reliability criteria used for this pilot study required no false positive and no false negative answers. The patients had an absence of retinal abnormalities (other than the nerve fibre layer defect) which might have contributed towards the field abnormalities. None of the patients was suffering from diabetes. They were evaluated according to the criteria of the Normal Tension Glaucoma Study Group ${ }^{13}$ : VF damage was indicated by (1) at least three points in cluster depressed 5 decibels or more from age corrected normal value, and (2) at least one of these points deviates more than 10 decibels from the normal value. The visual fields had to be reliable and the field defect was found in at least two of three consecutive VF examinations. Five of the 12 patients had a localised defect with early VF damage (MD $<5 \mathrm{~dB})$ corresponding to the site of the RNFL damage in the Humphrey 30-2 visual field. In seven eyes, the Humphrey 30-2 visual fields were repeatedly normal in spite of the presence of RNFL abnormalities. However, in five of these seven eyes, a paracentral visual field defect corresponding to the location of the RNFL defect was found when the central $8^{\circ}-10^{\circ}$ visual fields were examined with one degree resolution and with high pass resolution perimetry. The $10^{\circ}$ visual field was examined first using the Humphrey 10-2 off axis program and then with a user defined on axis $8^{\circ}$ visual field with $2^{\circ}$ resolution. ${ }^{7}$ High pass resolution perimetry was performed using the $30^{\circ}$ ring and $8^{\circ}$ centring programs of the Ophtimus perimeter (High Tech Vision, Malmö, Sweden). After pointwise comparison of the thresholds between the superior and inferior hemifields, a localised field defect was defined to be present if the thresholds of at least four points in cluster deviated 4 decibels or more from the corresponding point in the opposite hemifield. In all 10 eyes with visual field damage the visual field defect corresponded in location to the localised RNFL defect which was estimated in degrees from the fixation, when the distance between the optic disc and the macula was defined to be $15^{\circ}$. The definition of normality and abnormality of the high pass resolution visual fields included agreement of all four readers in a previous study. ${ }^{7}$ In two eyes the Humphrey 30-2,
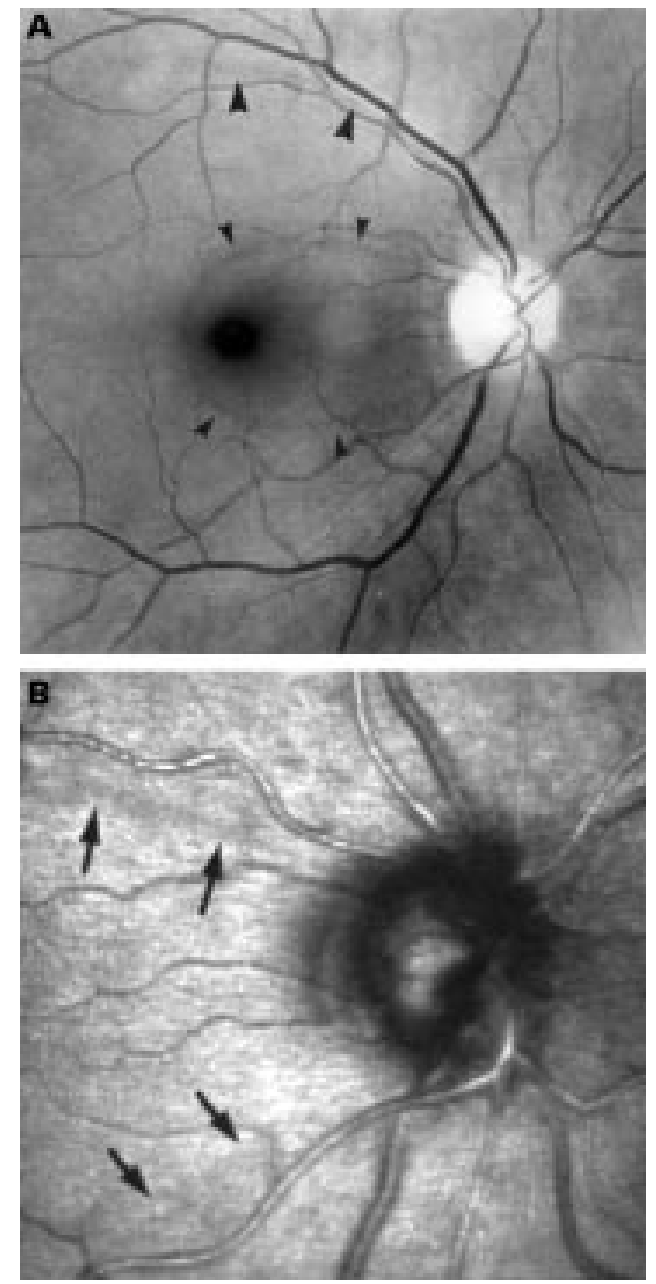

Figure 1 (A) A localised nerve fibre layer defect is present at 10'o clock and at 8'o clock in the RNFL photograph. No functional abnormalities were detected (patient no 3 . Table 1). (B) $15^{\circ}$ Laser scanning optical section image (patient no 3).

the $10^{\circ}$ visual fields, high pass resolution perimetry, and the optic disc appearance were normal with the localised RNFL defect as the only abnormality (Fig 1A). The normal clinical appearance of the optic disc as seen with the ophthalmoscope was judged according to the criteria of Hitchings and Spaeth. ${ }^{14}$ Four eyes had a history of increased intraocular pressure over $22 \mathrm{~mm} \mathrm{Hg}$, eight eyes were always normotensive - that is, the untreated intraocular pressure did not exceed $22 \mathrm{~mm} \mathrm{Hg}$.

From all patients who we selected to participate in the study and who met the requirements of the Declaration of Helsinki informed consent was obtained after the procedure had been fully explained.

LASER SCANNING TOMOGRAPHY: IMAGE ACQUISITION

In each eye, tomographic series of 32 optical section images were obtained from the parapapillary retina at the sites of the localised RNFL defects using laser scanning tomography (Heidelberg retina tomograph, Heidelberg Engineering, Heidelberg, Germany). ${ }^{15-17}$ The procedure applied in this study was as follows: with a $15^{\circ}$ image size (Fig $1 \mathrm{~B}$ ), the operator (RB) searched for the presence of a localised 

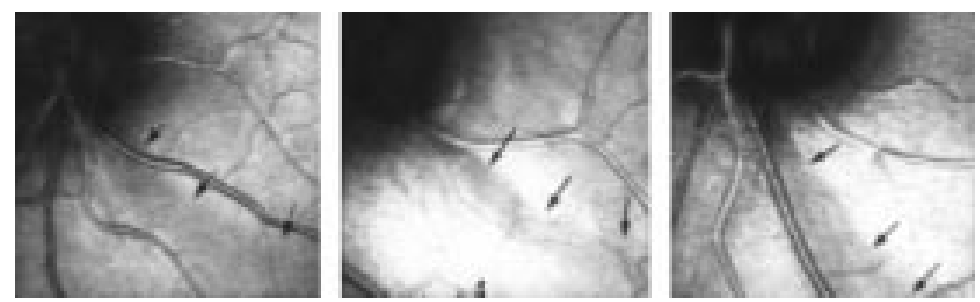

Figure 2 Localised RNFL defects in patients 4, 5, and 6 (Table 1) in the temporal inferior sector.

reduction of reflectivity due to localised RNFL defects by observing the live image in grey values on the monitor display of the instrument. The area of reflectivity reduction, which indicates the localised RNFL damage, was found predominantly near the temporal superior or even more pronounced near the inferior arcades (Fig 2). This area was centred within the image after the field of view had been reduced to $10^{\circ}$ The scanner was adjusted to a position where the raw images were equally illuminated on the right and left side of the live image ( $\mathrm{x}$ axis) and on the upper and lower border of the life image (y axis) to achieve correction for image tilt in both axes. An attempt was made to place the first focal plane within the posterior vitreous just anterior to the vitreoretinal interface as indicated by the first reflections of retinal vessels. The last focal plane was chosen near the site of the retinal pigment epithelium as indicated by a loss of reflectivity and visibility of retinal structure details due to a homogeneous absorption. In five eyes, the localised RNFL defects were present in the temporal inferior sector, in six eyes in the temporal superior sector. One patient had localised defects in both temporal sectors (Fig $1 \mathrm{~A}$ and $\mathrm{B})$.

IMAGE EVALUATION

The recorded tomographic images were evaluated as follows: each single optical section image, the calculated reflectivity image and the calculated topographic image were judged with respect to the visibility of areas of RNFL damage, indicated by a reduction of surface reflectivity.

All three observers judged the tomographic images for the visibility of RNFL damage. If
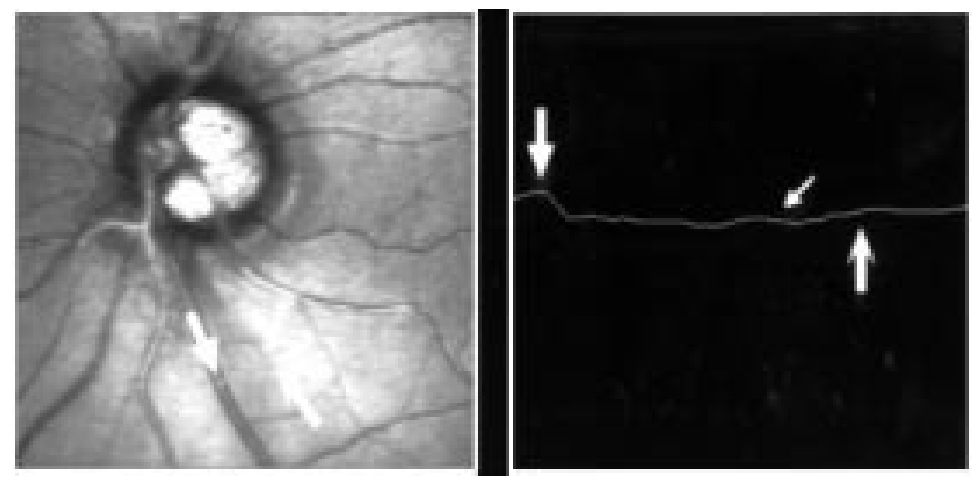

Figure 3 Determination of the width of the defect at $1 \mathrm{~mm}$ distance from the optic disc border. The profile cut displays the surface topography along the line drawn at the site of the RNFL defect. Arrows indicate the location of large and small vessels, the arrow with the curved head shows the upper border of the RNFL defect. one out of three observers could not identify a RNFL defect, the defect was classified as not visible.

The patients had been selected on the basis of the presence of detectable RNFL defects on photography. All investigators had agreed that localised RNFL defects could be detected on careful observation of the RNFL photographs. While the localised RNFL defects were easily seen during the confocal scanning laser imaging procedure on the real time monitor display of the device, the evaluation of the computed averaged images did not display RNFL defects which would be considered to be of significance for all three investigators in all cases, even with the prior knowledge of the site where areas of localised RNFL damage were to be expected. Two observers were unmasked to the visual field results (AT, JPA). One observer who performed the measurements (RB) was masked to the visual field results.

The width of the defect was measured at $1 \mathrm{~mm}$ distance from the border of the optic disc (Fig 3), corrected for corneal curvature and automatically for refractive error. ${ }^{16}$ The latter is obtained according to the focal plane position by the computer software. Based on the single optical section images which displayed the RNFL damage, the tomographic image series underwent further evaluation. A user defined program was developed to measure the mean surface height in the defective area-that is, the "area of interest", from approximately 1500 to 3500 voxels (volume elements) depending on the size of the defect. The term voxel indicates that the image subelements are defined in three dimensions (X, Y, and $\mathrm{Z}$ coordinates). To evaluate the reproducibility of the determination of mean height readings in the sample under investigation, the areas of interest were outlined and the average height positions were evaluated three times independently. In one patient with RNFL defects in both temporal segments, only the superior defect was randomly selected for the analysis.

We outlined the defective area interactively by drawing a contour line along the border of the RNFL defect in the optical section image which displayed the damage most clearly on the monitor display by means of a localised reduction of reflectivity. The optical section image was selected by the investigator who was masked for the visual field results. The section images were chosen according to the best contrast in reflectivity between the apparently normal retinal surface and the RNFL defect on the grey value display of the monitor. The mean height of the RNFL defect was measured by calculating the average of the $\mathrm{z}$ positions of all voxels within the outlined area (Fig 4, left). The mean height of the adjacent retina on both sides with normal surface reflectivity was measured in the same way (Fig 4, right). A difference between the mean height recordings in the defective and normal area which exceeded the twofold standard deviation of the mean height estimation was called a surface depression. All measurements were repeated three times. A prerequisite for the topographic 


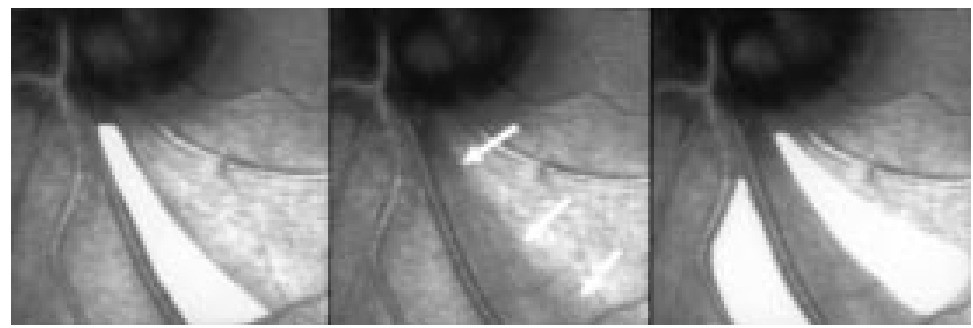

Figure $4 \quad 10^{\circ}$ Optical section image. Outline of the areas from which the average voxel height is calculated. Left: localised RNFL defect, right: adjacent retina with apparently normal reflectance.

analysis was a well shaped, single humped $\mathrm{z}$ profile of the intensity profile of the reflected light over the 32 optical section images. ${ }^{18}$ In addition, we measured the average reflectance at the site on the RNFL defect and the adjacent retina and calculated the ratio of these two measurements. This reflectance ratio gives the percentage of surface reflectivity at the site of the damaged area in comparison to the adjacent retina when multiplied by a factor of 100 .

\section{Results}

The results of the topometric analysis of the RNFL defects in regard to the visual field tests are summarised in Tables 1 and 2 .

\section{VISIBILITY OF RNFL DEFECTS}

RNFL defects were present in nine of 12 reflectivity images and were visible within single optical section images in all eyes (Tables 1 and 2). Visibility in reflectivity images was best when the reflectance ratio was approximately 0.8 or smaller. In contrast with the real time images of the monitor display during the image acquisition procedure, the defects were hardly seen in the recorded series if the reflectance ratio was $>0.94$.

WIDTH OF RNFL DEFECTS

The widths of the RNFL defects in the five eyes with abnormal Humphrey 30-2 program were $0.25 \mathrm{~mm}, 0.34 \mathrm{~mm}, 0.42 \mathrm{~mm}, 0.72 \mathrm{~mm}$,

Table 1 Eyes with surface depression at the site of RNFL damage. The RNFL defects were visible in selected single optical section images, in the reflectivity images, and in the topographic images. Given are patient number, age (years), perimetric method that detected visual field defects (visual field), the width of the defect at $1 \mathrm{~mm}$ distance from the optic disc border $(\mathrm{mm})$, the surface depression of the defect compared with the adjacent retina (depth: $\mu \mathrm{m}$ ), and the reflectance ratio (reflectivity in the damaged area/reflectivity in the normal retina)

\begin{tabular}{lllllll}
\hline Number & 1 & 2 & 3 & 4 & 5 & 6 \\
Age & 65 & 73 & 67 & 68 & 58 & 56 \\
Visual field & $30-2$ & Centring & none & $30-2$ & $10-2$ & $30-2$ \\
Width $(\mathrm{mm})$ & 0.25 & 0.20 & 0.11 & 0.34 & 0.28 & 0.42 \\
Depth $(\mu \mathrm{m})(\mathrm{SD})$ & $47(3)$ & $30(6)$ & $35(4)$ & $21(7)$ & $35(3)$ & $35(3)$ \\
Ratio & 0.93 & 0.91 & 0.94 & 0.80 & 0.81 & 0.68 \\
\hline
\end{tabular}

Table 2 Eyes without measurable surface depression at the site of RNFL damage. The RNFL defects were visible in selected single optical section images, but not in the topographic images. See legend of Table 1 for further information

\begin{tabular}{lllllll}
\hline Number & 7 & 8 & 9 & 10 & 11 & 12 \\
Age & 73 & 60 & 58 & 46 & 60 & 54 \\
Visual field & $30-2$ & $10-2$ & $30-2$ & Centring & $10-2$ & none \\
Width $(\mathrm{mm})$ & 1.0 & 0.25 & 0.72 & 0.16 & $0.10 \dagger$ & 0.18 \\
Depth $(\mu \mathrm{m})(\mathrm{SD})$ & $\star$ & $-5(5)$ & $-3(1)$ & $5(6)$ & $7(13)$ & $-1(6)$ \\
Ratio & 0.86 & 0.93 & 0.95 & 0.95 & 0.96 & 0.89 \\
\hline
\end{tabular}

${ }^{\star}$ Excluded from three dimensional topographic evaluation because of insufficient signal to noise ratio due to media opacities.

†The localised defect did not extend within $1 \mathrm{~mm}$ from the disc edge. It was therefore measured at a distance of $3.0 \mathrm{~mm}$ from the disc border. and $1.0 \mathrm{~mm}$ (mean 0.55 (SD 0.28) $\mathrm{mm}$ ). In the five eyes with abnormal Humphrey 10-2 or centring field, but normal Humphrey 30-2 visual fields the widths were $0.1 \mathrm{~mm}, 0.16 \mathrm{~mm}$, $0.20 \mathrm{~mm}, 0.25 \mathrm{~mm}$, and $0.28 \mathrm{~mm}(0.2(0.06)$ $\mathrm{mm})$. In two eyes in which all three methods of visual field testing were normal, the widths of the defects were $0.11 \mathrm{~mm}$ and $0.18 \mathrm{~mm}$.

DEPTH OF RNFL DEFECTS

Because of bad signal to noise ratio due to media opacities, depth measurements could not be evaluated in one eye (AR, Table 2 ). In the remaining 11 of 12 eyes, the image quality allowed for a three dimensional topographic analysis. ${ }^{19}$ The intraimage reproducibility of the mean height readings in triple measurements was 3.7 (SD 3.1) $\mu \mathrm{m}$ (range 0.8-10.2 $\mu \mathrm{m})$ for the site of the RNFL defect and 3.6 (1.5) $\mu \mathrm{m}(0.8-6.7 \mu \mathrm{m})$ for the areas of adjacent normal retina. Six of 11 eyes showed a height difference between the presumably normal and the defective retina of more than $20 \mu \mathrm{m}$. The mean surface depression was 34 (5) $\mu \mathrm{m}$ (21-47 $\mu \mathrm{m})$ at the site of the RNFL damage. The RNFL defects in these eyes were visible in the single optical section images, in the reflectivity images and in the topographic images. No significant surface depression was found in five eyes (mean height difference between the areas of interest $-0.6(1.5) \mu \mathrm{m} ;-5$ to $7 \mu \mathrm{m})$.

\section{Discussion}

Based on clinical observations in stereoscopic enlarged optic disc photographs it has been hypothesised that axon bundles originating in the mid peripheral fundus are located deep in the retina while more proximally originating fibres would be located more superficially, closer to the vitreous. ${ }^{1120}$ Such topographic organisation would, in fact, be the only one to explain why the degeneration of nerve fibres producing the early scotomas in the nasal visual field close to the horizontal meridian cannot be visualised and followed all the way to the edge of the optic disc. Furthermore, it could be seen from the stereophotographs that in locations where close to the optic disc a localised RNFL defect seemed to disappear under healthy looking axon bundles, there was no noticeable surface depression there. ${ }^{11}$

The results of the present study are in concert with these early clinical observations in showing that the degeneration of the deeply situated nerve fibres did not cause a measurable depression of the surface of the RNFL in all cases. These findings are not likely to be due to lack of resolution of the measuring device, but due to the factual existence of a topographic organisation of the RNFL postulated above.

The smallest defects (patients 8 to 12; Table 2) were seen only in single optical section images suggesting that they were located within the RNFL - that is, partial thickness defects, probably surrounded both by more peripherally originating fibres underneath the defect and more proximally originating fibres on top of the defect. 
In spite of the small sample size, there seemed to be a trend between the width of the RNFL defect and the visual field abnormalities. In the sample under investigation, all eyes with a scotoma in $6^{\circ}$ grid standard perimetry had an RNFL defect width of at least $0.25 \mathrm{~mm}$. The presence of retinal surface depressions could not be used to predict the outcome of the visual field test. The depth values did not differ between eyes with and without $6^{\circ}$ grid visual field damage (Tables 1 and 2).

The evaluation of the reflectance ratio from laser scanning tomograms is a new approach to quantify the clinically well known phenomenon of localised nerve fibre bundle defects, indicated by a localised reduction of surface reflectivity. The calculation of a ratio was chosen to compensate for the interindividual variability of light transmission through the optic media. RNFL defects were hard to find when the reflectance ratio was greater than 0.94 in the sample under investigation.

RNFL surface depression measurements may prove to be a good investigational tool to differentiate between "full thickness" and "partial thickness" retinal nerve fibre layer defects. The interest in the use of computerised image analysis techniques has increased over the past years especially in glaucoma research. Confocal scanning laser tomography was designed to evaluate the three dimensional structure of the optic disc by means of quantitative variables. Our aim was to use the potential of optical sectioning by confocal scanning laser tomography to evaluate retinal nerve fibre layer surface defects by quantitative variables For the topometric analysis of the optic disc, the optic nerve head is centred within the field of view. The scan depth is adjusted according to the cup depth. The technique which we applied for the present study differed considerably from the standard approach. The imaging procedure was started using a $15^{\circ}$ field of view, which was reduced to a $10^{\circ}$ viewing angle after the RNFL defect was imaged in the centre of the scanning laser beam. The overall scan depth was reduced to a maximum of $1.5 \mathrm{~mm}$. These efforts are necessary to use the full potential of confocal laser scanning tomography as a tool for quantitative RNFL evaluation.

The technique of image evaluation differed as well from the standard method to look at laser scanning tomograms of the optic nerve head. Normally, the averaged image is described by means of quantitative variables once a contour line has been drawn along the disc border on the monitor display of the instrument. For the assessment of the nerve fibre layer images, we evaluated not only the calculated reflectivity and topography images but inspected the single optical section images as well. This was necessary, because some of the RNFL defects which were seen on the monitor display in the real time image of the device were much harder to detect or were not detected at all in the calculated averaged images. Unless single optical section images are inspected, information regarding the RNFL may be lost when laser scanning tomo- grams are evaluated as usual for the optic disc analysis.

The findings which are reported in this paper demonstrate that further insight in the anatomical structure of the retinal nerve fibre layer topography might be gathered by in vivo investigations in the living human eye.

If we compare the use of the HRT with respect to nerve fibre layer imaging to conventional nerve fibre layer photography irrespective of the possibility to generate quantitative data, the confocal laser scanning system has both advantages and disadvantages. The laser wavelength of $670 \mathrm{~nm}$ in the near infrared range of the visible spectrum undergoes little absorption within the human lens in contrast with blue or green light. This is especially beneficial in cases of media opacities due to cataract formation in early stages.

A considerable disadvantage especially with regard to screening purposes is the small field of view which is limited to approximately $20^{\circ}$.

Longitudinal studies are currently being carried out to evaluate the mode of progression of localised RNFL defects with respect to surface reflectivity and defect depth. Because this is the first paper to describe the assessment of the three dimensional RNFL topography, it is not yet known whether localised retinal nerve fibre layer defects will simultaneously change in depth and width or whether these factors may show independent alterations.

Further studies will include the comparison of the new approach for RNFL assessment described in this paper with additional noninvasive diagnostic tools such as scanning laser polarimetry, scanning laser ellipsometry, and optical coherence tomography.

The study was supported in part by the Deutsche ForschungsThe study was supported in part by the Deutsche Forschungs-
gemeinschaft DFG Vö 437/1-2 (RB), the Griebels Foundation gemeinschaft DFG Vö 437/1-2 (RB), the Griebels Foundation
(RB), the Seiters Foundation (RB), the Juselius Foundation (AT), the Seiters Foundation (RB), the Jus the Paulo Foundation (AT; JA)

Proprietary interest: None.

1 Vogt A. Herstellung eines gelbblauen Lichtfiltrates, in welchem die Macula centralis in vivo in gelber Färbung erscheint, die Nervenfasern der Netzhaut und andere feine Einzelheiten derselben sichtbar werden, und der Grad der Gelbfärbung der Linse ophthalmoskopisch nachweisbar Gelbfärbung der Linse ophthalmoskopisch

ist. Graefes Arch Ophthalmol 1913;84:293-311.
2 Hoyt WF, Newman NM. The earliest observable defect in glaucoma? Lancet 1972;1:692-3.

3 Sommer A, Quigley HA, Robin AL. Evaluation of nerve fiber layer assessment. Arch Ophthalmol 1984;102:1766-71.

4 Tuulonen A, Airaksinen PJ. Initial glaucomatous optic disc and retinal nerve fiber layer abnormalities and their progression. Am f Ophthalmol 1991;111:485-90.

5 Quigley HA, Katz J, Derick RJ, et al. An evaluation of optic disc and nerve fiber layer examinations in monitoring progression of early glaucoma damage. Ophthalmology 1992; 99:19-28.

6 Sommer A, Katz J, Quickley HA, et al. Clinically detectable nerve fiber layer atrophy precedes the onset of glaucomatous visual field loss. Arch Ophthalmol 1991;109:77-83.

7 Tuulonen A, Lehtola J, Airaksinen PJ. Nerve fiber layer defects with normal visual fields. Do normal optic disc and visual fields indicate absence of glaucomatos abnormality? Ophthalmology 1993;100:587-98.

8 Airaksinen PJ, Drance SM, Douglas GR, et al. Diffuse and localized nerve fiber loss in glaucoma. Am $\mathcal{F}$ Ophthalmol 1984;98:566-71.

9 Jonas JB, Nguyen NX, Naumann GOH. The retinal nerve fiber layer in normal eyes. Ophthalmology 1989;96:627-32.

10 Quigley HA, Reacher M, Katz J, et al. Quantitative grading system of nerve fiber layer photographs. Ophthalmology 1993;100:1800-7.

11 Airaksinen PJ, Alanko HI. Effect of retinal nerve fibre loss on the optic nerve head configuration in early glaucoma. on the optic nerve head configuration in early gla
Graefes Arch Clin Exp Ophthalmol 1983;220:193-6.

12 Airaksinen PJ, Nieminen H. Retinal nerve fibre layer photography in glaucoma. Ophthalmology 1985;92:877-9. 
13 Schulzer M, for the Normal Tension Glaucoma Study Group. Intraocular pressure reduction in normal-tension Group. Intraocular pressure reduction in normal-te
glaucoma patients. Ophthalmology 1992;99:1468-70.

14 Hitchings RA, Spaeth GL. The optic disc in glaucoma. I Classification. Br F Ophthalmol 1976;60:778-85.

15 Burk ROW, Rohrschneider K, Völcker HE, et al. Analysis of three-dimensional optic disk topography by laser scanning tomography. In: Nasemann JE, Burk ROW, eds. Laser scanning ophthalmoscopy and tomography. Munich: Quintessenz, 1990:161-76.

16 Rohrschneider K, Burk ROW, et al. Reproducibility of the optic disc nerve head topography with a new laser 1044-9.
17 Burk ROW, Völcker HE. Scanning laser ophthalmoscopy and tomography. In: Jay B, Kirkness CM, eds. Recent advances in ophthalmology. Vol 9. Edinburgh: Churchill 995:225-43

18 Zinser G, Harbarth U, Schröder H. Formation and analysis of three-dimensional data with the laser tomographic scanner LTS. In: Nasemann JE, Burk ROW, eds. Laser scanning ophthalmoscopy and tomography. Munich: Quintessenz, 1990:243-52

19 Weinreb RN, Lusky M, Bartsch DU, et al. Effect of repetitive imaging on topographic measurements of the optic nerve head. Arch Ophthalmol 1993;111:636-8. 20 Minckler DS. The organization of nerve fiber bundles in the
primate optic nerve head. Arch Ophthalmol 1980;98:1630-6. 